\title{
Cerebrospinal fluid lactate concentration to distinguish bacterial from aseptic meningitis: a systemic review and meta-analysis
}

\author{
Nguyen T Huy ${ }^{1}$, Nguyen TH Thao ${ }^{2}$, Doan TN Diep ${ }^{2,3}$, Mihoko Kikuchi ${ }^{1,4}$, Javier Zamora ${ }^{5}$, Kenji Hirayama ${ }^{1,4,6^{*}}$
}

\begin{abstract}
Introduction: Making a differential diagnosis between bacterial meningitis and aseptic meningitis is a critical clinical problem. The utility of a cerebrospinal fluid (CSF) lactate assay for this purpose has been debated and is not yet routinely clinically performed. To adequately evaluate this assay, a systematic review and meta-analysis of studies of the CSF lactate concentration as a marker for both bacterial meningitis and aseptic meningitis was performed.

Methods: Electronic searches in PubMed, Scopus, the MEDION database and the Cochrane Library were conducted to identify relevant articles published before March 2009. A manual search of reference lists from selected articles was also conducted. Two reviewers independently selected relevant articles and extracted data on study characteristics, quality and accuracy.

Results: Twenty-five articles were identified that met the eligibility criteria. Diagnostic odds ratios were considerably homogenous (Chi-square $P=0.1009, P^{2}=27.6 \%$ ), and the homogeneity was further confirmed by a Galbraith plot and meta-regression analysis using several covariates. The symmetrical summary receiver-operator characteristic curve (SROC), fitted using the Moses-Shapiro-Littenberg method, was positioned near the upper left corner of the SROC curve. The Q value and area under the curve were 0.9451 and 0.9840 , respectively, indicating excellent accuracy. The diagnostic accuracy of the CSF lactate concentration was higher than those of other four conventional markers (CSF glucose, CSF/plasma glucose quotient, CSF protein, and CSF total number of leukocytes) using a head to head meta-analysis of the 25 included studies.
\end{abstract}

Conclusions: To distinguish bacterial meningitis from aseptic meningitis, CSF lactate is a good single indicator and a better marker compared to other conventional markers.

\section{Introduction}

Accurate and rapid diagnosis of acute bacterial meningitis $(\mathrm{BM})$ is essential because disease outcome depends on immediate initiation of appropriate antibiotic therapy [1]. BM should be treated promptly with antibiotics, whereas acute aseptic meningitis (AM) is usually self limiting. However, differentiating BM from AM may be challenging for clinicians because the symptoms and laboratory assays are often similar and overlapping. In addition, classical clinical manifestations of BM in infants and children are usually difficult to recognize because of the absence of signs of meningeal irritation and because of delayed

\footnotetext{
* Correspondence: hiraken@nagasaki-u.ac.jp

'Department of Immunogenetics, Institute of Tropical Medicine (NEKKEN)

Nagasaki University, 1-12-4 Sakamoto, Nagasaki 852-8523, Japan

Full list of author information is available at the end of the article
}

elevation of intracranial pressure. Parameters examined in cerebrospinal fluid (CSF) are less descriptive in children than in adults: in enterovirus meningitis, CSF parameters can be practically identical to those of bacterial meningitis. For example, acute meningitis with predominance of neutrophils in CSF suggests BM; however, herpes simplex-1 infected meningitis presents with $>90 \%$ neutrophils in CSF [2]. Furthermore, other assays, such as Gram stain, latex agglutination, and polymerase chain reaction-based assays, lack sensitivity [3-6]. In practice, before definitive CSF bacterial cultures are available, most patients with acute meningitis are treated with broadspectrum antibiotics targeting BM. In general, this does not seriously harm the AM patient; however, it may enhance the local frequency of antibiotic resistance [7] and cause antibiotic adverse effects, nosocomial infections

\section{C) Biomed Central}


[8], and high medical costs [9]. Thus, it is not only important to recognize BM patients who promptly need antimicrobial therapy but also AM patients who do not need antibiotics and/or hospital stays.

In recent years, it has been proposed that CSF lactate may be a good marker that can differentiate bacterial meningitis $(>6 \mathrm{mmol} / \mathrm{l})$, from partially treated meningitis (4 to $6 \mathrm{mmol} / \mathrm{l})$ and aseptic meningitis $(<2 \mathrm{mmol} / \mathrm{l})$ [10]. However, other researchers have suggested that CSF lactate offers no additional clinically useful information over conventional CSF markers [11,12]. Other markers, such as C-reactive protein (CRP) [13] and procalcitonin [14], may allow differentiation of patients with bacterial meningitis from those with aseptic meningitis. However, neither of these markers is routinely used in clinical practice [4]. The reported diagnostic accuracy of CSF lactate for the differential diagnosis of BM from AM has varied across studies $[11,12]$. To adequately evaluate its accuracy, a systematic review and meta-analysis were performed on studies that had investigated the CSF lactate concentration as a differential marker in both BM and AM patients.

\section{Materials and methods}

A protocol was designed before this study was performed as recommended by the Quality of Reporting of Meta-analyses (QUORUM) statement [15] and the PRISMA Statement [16].

\section{Search strategy and study selection}

Four electronic databases, PubMed [17], Scopus [18], MEDION database [19] and the Cochrane Library [20], were searched for suitable studies published before March 2009. The search terms that were used included "meningitis AND (lactate OR lactic)". Only articles written in English that evaluated the CSF lactate/lactic acid concentration for differential diagnosis distinguishing BM from AM were included.

Clinical diagnosis was used as reference standard for $\mathrm{BM}$ and $\mathrm{AM}$ to avoid misclassification of BM patients as AM. For sub-group analysis, diagnosed BM was defined as a patient with CSF pleocytosis (CSF leukocyte count > 4 cells/ $\mu \mathrm{l}$ ) and one of the following criteria: (1) positive CSF Gram-stained smear for a bacterial pathogen, (2) positive CSF culture for a bacterial pathogen, (3) positive CSF latex agglutination assay or polymerase chain reaction assay for a bacterial pathogen, or (4) positive blood culture. Diagnosed viral AM was defined as the diagnosis of a patient with pleocytosis in the CSF of $\geq 4$ leukocytes/ $\mu \mathrm{l}$ combined with the absence of any of the four criteria for BM and with either of the following criteria: a positive polymerase chain reaction assay or a positive culture for viral pathogen or specific antiviral antibodies in CSF and serum [21].
Studies with fewer than 16 participants were excluded in order to limit selection bias ( $\geq 8 \mathrm{BM}$ patients and $\geq 8 \mathrm{AM}$ patients were required for inclusion) [22]. Furthermore, the following studies were also excluded: (1) animal studies, case reports, replies and reviews; (2) studies in which data could not be extracted; and (3) studies that used lactate as a criteria for diagnosis of AM.

Two independent reviewers (NTH and NTHT) scanned primary titles and abstracts (when available) to select potential full text articles for further scrutiny. When the title and abstract could not be rejected by any reviewer, the full text of the article was obtained and carefully reviewed for inclusion by the two reviewers. Inclusion or exclusion of each study was determined by discussion and consensus between the two reviewers. If multiple reports contained overlapping cases, only the largest report was included. When overlap could not be determined conclusively, the study with the most inclusive information or the latest report was included.

\section{Data extraction}

Two independent investigators (NTH and NTHT) extracted data from the studies chosen for inclusion. Disagreements were resolved by discussion and consensus. Studies with criteria for establishing the diagnosis of BM that relied solely on clinical or laboratory improvement after antibiotic therapy were excluded. In selected studies, the following patients who met the following criteria were also excluded from the BM groups: (1) patients with tuberculous or fungal meningitis, (2) BM patients who received antibiotics before lumbar puncture, (3) post-surgery or traumatic patients, and (4) patients with other central nervous system conditions that could contribute to elevation of CSF lactate (such as recent stroke, seizures, brain hypoxia, and brain trauma). A $2 \times 2$ diagnostic table was constructed from informative descriptions, lactate values, lactate plots, sensitivity, specificity, likelihood ratios, and receiveroperator characteristic (ROC) curves. Other information for each study, such as author, publication year, age range of patients, assay methods, stabilizer addition versus immediate measurement of lactate, prior antibiotic treatment, tuberculosis, country and city where the study was performed, study design (cross sectional or case control), data collection (prospective or retrospective), assignment of the patient (consecutive or random), and blinded interpretation of lactate measurements and diagnostic results, were also recorded.

\section{Quality assessment}

The quality of included studies was assessed using criteria suggested by Pai et al. [23], as it has been observed that these criteria can affect the accuracy of the lactate 
method. The quality of each study included in the metaanalysis was determined across five metrics: diagnostic criteria, study design, exclusion of patients who received antibiotics before lumbar puncture, exclusion of patients with other disorders, and the method of the lactate assay. Since case-control studies reportedly overestimate the accuracy result [24], the study design was scored as follows: studies with cross-sectional were assigned one point; those with case-control were assigned zero points. For data collection, prospective studies were identified and assigned two points, retrospective studies were assigned one point, and a study with unknown study design was assigned zero points. In addition, studies that recruited consecutive or random patients were assigned one point, while studies without this kind of information were assigned zero points. Studies excluding chronic diseases or other central nervous disorders patients were assigned one point. Studies that originally excluded data from subjects who received antibacterial therapy prior to lumbar puncture were assigned two points, while studies that included subjects who received antibacterial therapy prior to lumbar puncture and excluded in the present report were assigned one point. Studies that originally excluded data from subjects with TB meningitis were assigned two points, while studies that included these subjects and were excluded by us in this report were assigned one point. For the quality of the method, studies with blinded assessment of the lactate assay with diagnostic results were assigned one point. Since sample processing is another important issue that may affect the accuracy of the assay [25], studies using a stabilizer for lactate sample processing or measuring immediately were assigned one point. Quality was evaluated by discussion and consensus after the independent review of each study by two authors (NTH and NTHT).

\section{Meta-analysis}

Data were analyzed using Meta-Disc (version 1.4) software (Unit of Clinical Biostatistics, Ramón y Cajal Hospital, Madrid, Spain) [26] unless otherwise stated. The software is publicly available [27]. Accuracy measures including sensitivity, specificity, positive likelihood ratio (LR+), negative likelihood ratio (LR-), and diagnostic odds ratio (DOR) were computed. The DOR describes the ratio of the odds of a positive assay in a BM patient compared with a AM patient and was calculated by LR+/LR- (or (sensitivity/(1-specificity))/((1-sensitivity)/specificity)) [28]. A DOR $>1$ indicated the assay had discriminative power; a higher DOR indicated more discriminative power.

Heterogeneity of both the sensitivity and specificity across the studies was tested using a $\chi^{2}$ test. A $\chi^{2}$ $P$-value of $<0.05$ was considered heterogeneous. An alternative method to explore the heterogeneity, the $I^{2}$ index, was also used. The $I^{2}$ index presents the percentage of total variation across studies that is due to heterogeneity rather than chance [29]. $I^{2}$ values of $>25 \%$, $50 \%$, or $75 \%$ were considered to reflect low, moderate, and high heterogeneity, respectively [29].

Pooling of data was performed if sensitivity and specificity were homogeneous [22]. In the case of heterogeneity, a Spearman rank correlation coefficient $(\rho)$ was calculated to measure the extent of correlation between sensitivity and specificity. With the Spearman rank correlation coefficient, if there is a correlation the variation between studies is mainly due to different cut-off values and a summary receiver operating characteristic curve may be modeled [22]. A symmetrical SROC fitting was performed when the DOR was found to be constant. A constant DOR is equivalent to the slope of the fitted regression line at zero (testing whether parameter $b=0$ ) [26]. As the natural log of DOR (lnDOR) reflects heterogeneity, heterogeneity was explored by subgroup analysis [22]. This subgroup analysis was performed using a univariate meta-regression analysis in order to evaluate the effect of covariates on diagnostic accuracy (DOR). A Galbraith plot was constructed to further visually assess the heterogeneity of $\ln \mathrm{DOR}$ and to identify outlier studies [30]. For each study, the ratio of $\operatorname{lnDOR} /$ standard error (SE) of the $\operatorname{lnDOR}(\mathrm{SE}(\operatorname{lnDOR}))$ was plotted against $1 / \mathrm{SE}(\mathrm{lnDOR})$, and was represented by a single dot [22]. If the heterogeneity of $\operatorname{lnDOR}$ remained between studies, the DerSimonian-Laird random effects model (REM) for fitting SROC was chosen [22], and a $P$-value $<0.05$ was considered significant. In addition, the heterogeneity of $\ln$ DOR across studies was also examined using multivariable logistic meta-regression analysis with the following covariates as predictor variables: criteria for AM, study design (prospective or retrospective), patient recruitment methods (consecutive or random), assay methods, exclusion criteria, prior antibiotic treatment, tuberculous (TB) meningitis, blinded interpretation of lactate measurement, reliability of the method (stabilizer for lactate sample or immediate measurement), quality assessment score, cut-off points, lactate method, age of participants (child or adult), total number of participants, and effective sample size (ESS) (where ESS $=\left(4 n_{1 *} n_{2}\right) /\left(n_{1}+n_{2}\right)$ ) [31]. The variable with the highest $P$-value was excluded from the subsequent round of analysis in the multivariable meta-regression model in a stepwise downward manner. A variable was kept in the model if $P$-value < 0.05 . The beta-coefficients and corresponding relative DOR from the meta-regression analysis revealed the effect of each variable on the DOR. If a variable was strongly associated with accuracy, further analysis within sub-groups (with a minimum of three studies per subgroup) was conducted to determine diagnostic accuracy and its SROCs. 
To further evaluate the accuracy of the CSF lactate concentration, the $\mathrm{Q}$ value and area under the curve (AUC) were calculated from the SROC curves. The Q value is the intersection point of the SROC curve with a diagonal line of the ROC space at which sensitivity equals specificity; a higher $\mathrm{Q}$ value indicates higher accuracy. AUC values $\geq 0.5,0.75,0.93$, or 0.97 were considered to represent fair, good, very good, or excellent accuracy [32].

\section{Publication bias}

Since publication bias is a concern for meta-analysis, the potential presence of this bias was identified using a funnel plot and Egger test [33]. If publication bias was found, the trim and fill method of Duvall and Tweedie was performed to add studies that appeared to be missing $[34,35]$ using the Comprehensive Meta-analysis software version 2.0 (Biostat Inc. Englewood, NJ, USA) [36]. The pooled DOR and its 95\% confidence interval were adjusted after the addition of potential missing studies.

\section{Results}

\section{Literature search}

The literature search initially identified 447 and 600 publications from Pubmed and Scopus, respectively (Figure 1). After an initial screening of the title and/or abstract, 115 articles were included for full text reading. Then additional studies were identified by searching reference lists and articles that cited relevant publications using Scopus databases from full text reviews, review articles, and textbook chapters. These titles and abstracts were reviewed, and the full text was read if necessary. A total of 90 articles were excluded from final analysis due to the following reasons: (1) comment/review/guidelines/reply/case report $(n=22)$, (2)non-English language $(n=1)$, (3) no lactate concentration $(n=7)$, (4) no BM or AM group $(n=20)$, (5) in vitro or animal research $(n=3),(6)$ unable to exclude partially treated patients $(n=6),(7)$ unable to extract data $(n=11)$, and (8) low number of participants $(n=$ $20)$. Finally, 25 studies were selected for final analysis $[11,12,37-58]$ with agreement between the two reviewers $(\kappa=0.898)$.

The 25 selected publications, which were performed in 16 countries and on five continents, included $783 \mathrm{BM}$ and 909 AM patients. The characteristics of these studies are outlined in Table 1. The average sample size of the included studies was 31 patients (range, 11 to 86) for the BM group and 36 patients (range, 9 to 128) for the AM group. A total of three different methods for lactate measurement (enzymatic: $n=19$, automatic analyzer: $n=2$, gas-liquid chromatography $n=2$ ) were performed in the 25 included studies. One study used both enzymatic and gas-liquid chromatography methods, with consistent results between the analysis techniques. In all of the 25 included studies, the cut-off value of CSF lactate of $<3.5 \mathrm{mmol} / \mathrm{L}$ was applied in 12 studies, while the cut-off value of $\geq 3.5 \mathrm{mmol} / \mathrm{L}$ was applied in $12 \mathrm{stu}$ dies. One study did not indicate the CSF lactate concentration cut-off value.

\section{Quality of selected studies}

In all of the 25 included studies, the lactate assay did not play a role in the final diagnosis of BM or AM. For the study design, 18 studies (72\%) were cross-sectional, while seven studies (18\%) were case-control studies or not reported (Table 2). Concerning study design, five (21\%) collected data prospectively, three $(13 \%)$ collected data retrospectively, and 16 (69\%) did not report the study design. Twelve (50\%) studies used either consecutive or random recruitment of participants, while the remaining studies $(50 \%)$ did not state the method of participant selection. Only one study (4\%) described exclusion criteria for participant enrolment, which included the exclusion of patients with chronic diseases or central nervous system disorders. Eleven studies (46\%) did not include data from patients who received antibacterial therapy prior to lumbar puncture, seven studies $(30 \%)$ enrolled subjects who received antibacterial therapy prior to lumbar puncture (these data were excluded in the present report), and six studies (26\%) did not mention prior antibacterial therapy. Fourteen studies (58\%) originally excluded data from subjects with tuberculous meningitis; eight studies (35\%) included these subjects and were excluded in the present study, while no such information could be found in two studies (9\%). Concerning the quality of the lactate method, a blinded assessment of the lactate assay with diagnostic results was reported in only three studies $(13 \%)$, while a stabilizer was used for the lactate sample or an immediate lactate measurement was described in 13 (54\%). No study scored the maximal points (11) in the present analysis, while one study received one point. The range of total points was one to eight (Table 2).

\section{Meta-analysis}

The sensitivity of included studies ranged from 0.86 to 1.00 (mean, 0.96; 95\% confidence interval (CI), 0.95 to 0.98 ) (Figure 2), while the specificity varied widely from 0.43 to 1.00 (mean, $0.94 ; 95 \% \mathrm{CI}, 0.93$ to 0.96 ). The mean of LR+ was calculated at 14.53 (95\% CI, 8.07 to 26.19), LR- at 0.07 (95\% CI, 0.05 to 0.09 ) and the mean DOR was 270.0 (95\% CI, 142.54 to 519.04 ).

Heterogeneity was present among the studies with regard to specificity $\left(\chi^{2} P=0.000, I^{2}=73.6 \%\right)$, and to LR+ $\left(\chi^{2} P=0.000, I^{2}=79.5 \%\right)$. Therefore, pooling of data was not performed [22]. Because of the significant heterogeneity of these data, the Spearman rank correlation coefficient 


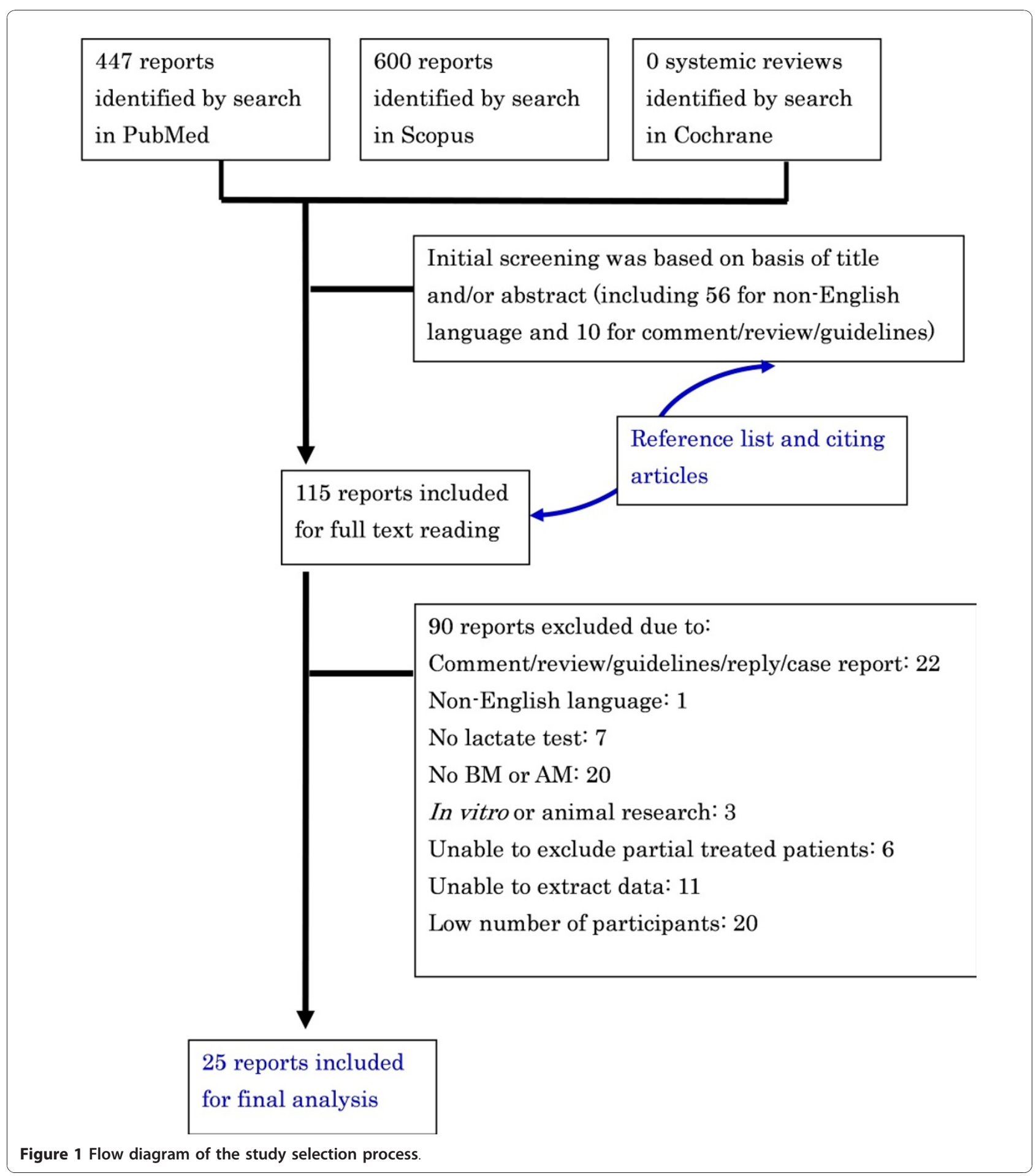

$(\rho)$ was calculated to measure the extent of correlation between sensitivity and specificity. The present results indicated a poor correlation between sensitivity and specificity, with a Spearman $P=-0.043$, suggesting that variation between studies was not mainly due to different cut-off values [22]. In contrast, homogeneity was present among the studies with regard to sensitivity $\left(\chi^{2} P=0.12\right.$, $\left.I^{2}=25.9 \%\right)$, LR- $\left(\chi^{2} P=0.66, I^{2}=0.0 \%\right)$, and for DOR $\left(\chi^{2}\right.$ $\left.P=0.1009, I^{2}=27.6 \%\right)$. A Galbraith plot was created to graphically assess the homogenous nature of the $\operatorname{lnDOR}$, and to identify potential outlier studies (Figure 3). On the Galbraith plot, 24 studies were inside the $95 \%$ bounds 
Table 1 Summary of included studies

\begin{tabular}{|c|c|c|c|c|c|c|c|c|c|c|c|}
\hline \multirow[t]{2}{*}{ Study (ref) } & \multirow[t]{2}{*}{ Year } & \multirow[t]{2}{*}{ Country } & \multicolumn{2}{|c|}{ Number of patients } & \multirow[t]{2}{*}{ Age } & \multirow[t]{2}{*}{ Lactate method } & \multirow[t]{2}{*}{ Cut-off (mmol/L) } & \multicolumn{4}{|c|}{ Test results } \\
\hline & & & BM & $A M$ & & & & $T P^{a}$ & FP & FN & TN \\
\hline Abro [37] & 2008 & UAE & 86 & 48 & Adult & $E n z^{d}$ & 3.8 & 85 & 0 & 1 & 48 \\
\hline Kleine [59] & 2003 & Germany & 73 & 128 & Adult & Enz & 2.61 & 73 & 0 & 0 & 128 \\
\hline Schwarz [58] & 2000 & Germany & 16 & 14 & Adult & $N R^{c}$ & 2.1 & 15 & 8 & 1 & 6 \\
\hline Uduman [57] & 2000 & UAE & 23 & 42 & Children & Enz & NR & 22 & 3 & 1 & 39 \\
\hline Cameron [38] & 1993 & UK & 11 & 9 & Children & Enz & 4.1 & 11 & 0 & 0 & 9 \\
\hline Genton [39] & 1990 & Switzerland & 19 & 28 & Adult & Auto $^{e}$ & 4.2 & 18 & 0 & 1 & 28 \\
\hline Shaltout [40] & 1989 & Kuwait & 14 & 9 & Children & Auto & 3 & 13 & 0 & 1 & 9 \\
\hline Donald [41] & 1986 & S. Africa & 43 & 23 & Children & Enz & 2.85 & 40 & 0 & 3 & 23 \\
\hline Nelson [42] & 1986 & Sweden & 11 & 28 & Children & Enz & 2.4 & 11 & 3 & 0 & 25 \\
\hline Low $[43]$ & 1986 & Singapore & 22 & 54 & Children & Enz & 2.78 & 19 & 8 & 3 & 46 \\
\hline Ruuskanen [12] & 1985 & Finland & 32 & 30 & Children & Enz & 3 & 30 & 2 & 2 & 28 \\
\hline Lester [44] & 1985 & Denmark & 15 & 15 & Child/adult & Enz & 4.3 & 15 & 0 & 0 & 15 \\
\hline Vanprapar [45] & 1983 & Thailand & 22 & 18 & Children & Enz & 3.89 & 20 & 0 & 2 & 18 \\
\hline Mandal [46] & 1983 & UK & 20 & 59 & Children & Enz & 3.9 & 20 & 5 & 0 & 54 \\
\hline Pönkä [47] & 1983 & Finland & 11 & 27 & Child/adult & Enz & 3 & 10 & 1 & 1 & 26 \\
\hline Briem [48] & 1983 & Sweden & 45 & 102 & Child/adult & Enz & 3.5 & 45 & 4 & 0 & 98 \\
\hline Berg [49] & 1982 & Sweden & 18 & 121 & Child/adult & Enz & 3 & 16 & 9 & 2 & 112 \\
\hline Eross [50] & 1981 & Australia & 66 & 31 & Child/adult & Enz & 3.9 & 64 & 0 & 2 & 31 \\
\hline Knight [51] & 1981 & US & 68 & 20 & Children & Enz & 3.3 & 68 & 3 & 0 & 17 \\
\hline Curtis [52] & 1981 & UK & 13 & 12 & Child/adult & Enz & 2.8 & 13 & 0 & 0 & 12 \\
\hline Lannigan [53] & 1980 & Canada & 14 & 14 & Adult & Enz & 3.89 & 13 & 3 & 1 & 11 \\
\hline Gästrin [11] & 1979 & Sweden & 38 & 17 & Child/adult & $G L^{b}$ & 3.5 & 37 & 3 & 1 & 14 \\
\hline Lauwers [54] & 1978 & Belgium & 35 & 20 & $N R^{c}$ & $\mathrm{GL}$ & 3.89 & 33 & 0 & 2 & 20 \\
\hline Controni [55] & 1977 & US & 55 & 15 & Children & Enz\&GL & 2.78 & 53 & 0 & 2 & 15 \\
\hline Bland [56] & 1974 & US & 13 & 25 & Children & Enz & 4.44 & 12 & 0 & 1 & 25 \\
\hline
\end{tabular}

${ }^{\mathrm{a}} \mathrm{TP}$, true-positive; FP, false-positive; FN, false-negative; TN, true-negative; ${ }^{\mathrm{b}} \mathrm{GL}$, gas-liquid chromatography; ${ }^{\mathrm{C}} \mathrm{NR}$, not reported; ${ }^{\mathrm{d}} \mathrm{Enz}, \mathrm{Enzymatic} ;{ }^{\mathrm{e}} \mathrm{Automatic}$ analyzer.

(the zones of two outer parallel lines drawn at two units over and below the regression) from the standardized mean $\operatorname{lnDOR}$, while only one study was the outlier [58]. However, the DOR was just slightly increased from 270.0 to 292.71 after removing the outlier study. further confirming the relatively homogenous nature of the $\ln D O R$ [22]. The homogenous nature of the $\ln D O R$ across studies was also examined using meta-regression analysis with the following covariates as predictor variables: data collection, study design (prospective or retrospective), recruitment of the patient (consecutive or random), assay methods, exclusion criteria, prior antibiotic treatment, tuberculous meningitis, blinded interpretation of lactate measurement, reliability of the method (lactate sample stabilizer or immediate measurement), quality assessment score, cutoff points, lactate method, age of participants (children/ adult), total number of participants, and effective sample size (ESS). The present results revealed an independent association of the $\operatorname{lnDOR}$ with tested covariates (Data not shown). These data suggest that the InDOR of the included studies is homogenous, and thus a SROC can be fitted based on the pairs of sensitivity and specificity of the individual studies [22].

The slope of the fitted regression line of the MosesShapiro-Littenberg model was zero (testing whether parameter $b=0, P=0.84)$, indicating a constant DOR. Therefore, a symmetrical SROC fitting was performed (Figure 4). The present results showed that the SROC curve was positioned near the upper left corner of the SROC curve, with the Q value and AUC at 0.9451 and 0.9840, respectively, indicating excellent accuracy.

\section{Sub meta-analysis of lactate as a differential marker for diagnosed BM from AM}

Meta-analysis was further performed to assess the diagnostic accuracy of lactate between diagnosed BM and AM. Nineteen studies [11,12,38,39,41-43,46-56,59] that analyzed only diagnosed BM and five other studies $[37,40,44,45,57]$ that included diagnosed BM as well as clinical BM that could be extracted separately were included in the subgroup analysis. The specificity and $\mathrm{LR}+$ were heterogeneous among the studies, but 
Table 2 Quality of included studies

\begin{tabular}{|c|c|c|c|c|c|c|c|c|c|}
\hline Study (ref) & Design $^{a}$ & Data collection $^{b}$ & Recruit $^{c}$ & Exclusion $^{d}$ & Prior treatment ${ }^{\mathrm{e}}$ & $T B^{f}$ & Blinded $^{\mathrm{g}}$ & Reliability ${ }^{h}$ & Total score \\
\hline Abro [37] & 0 & 0 & 0 & 1 & 2 & 2 & 0 & 0 & 5 \\
\hline Kleine [59] & 1 & 1 & 0 & 0 & 2 & 2 & 0 & 0 & 6 \\
\hline Schwarz [58] & 1 & 2 & 1 & 0 & 2 & 0 & 0 & 0 & 6 \\
\hline Uduman [57] & 1 & 2 & 1 & 0 & 2 & 2 & 0 & 0 & 8 \\
\hline Cameron [38] & 0 & 0 & 0 & 0 & 2 & 1 & 0 & 1 & 4 \\
\hline Genton [39] & 1 & 1 & 1 & 0 & 1 & 1 & 1 & 1 & 7 \\
\hline Shaltout [40] & 1 & 0 & 1 & 0 & 1 & 1 & 1 & 0 & 5 \\
\hline Donald [41] & 0 & 0 & 0 & 0 & 0 & 1 & 0 & 1 & 2 \\
\hline Nelson [42] & 1 & 1 & 1 & 0 & 1 & 2 & 1 & 1 & 8 \\
\hline Low $[43]$ & 0 & 0 & 0 & 0 & 1 & 2 & 0 & 0 & 3 \\
\hline Ruuskanen [12] & 1 & 0 & 0 & 0 & 0 & 0 & 0 & 0 & 1 \\
\hline Lester $[44]$ & 1 & 2 & 1 & 0 & 1 & 2 & 0 & 1 & 8 \\
\hline Vanprapar [45] & 1 & 0 & 0 & 0 & 0 & 0 & 0 & 0 & 1 \\
\hline Mandal [46] & 1 & 0 & 1 & 0 & 1 & 2 & 0 & 1 & 6 \\
\hline Pönkä [47] & 1 & 0 & 1 & 0 & 0 & 2 & 0 & 0 & 4 \\
\hline Briem [48] & 0 & 2 & 0 & 0 & 2 & 1 & 0 & 0 & 5 \\
\hline Berg [49] & 1 & 2 & 1 & 0 & 2 & 1 & 0 & 1 & 8 \\
\hline Eross [50] & 1 & 2 & 0 & 0 & 2 & 2 & 0 & 1 & 8 \\
\hline Knight [51] & 0 & 0 & 0 & 0 & 0 & 2 & 0 & 1 & 3 \\
\hline Curtis [52] & 1 & 0 & 1 & 0 & 2 & 1 & 0 & 1 & 6 \\
\hline Lannigan [53] & 1 & 0 & 1 & 0 & 2 & 2 & 0 & 0 & 6 \\
\hline Gästrin [11] & 1 & 0 & 0 & 0 & 0 & 2 & 0 & 1 & 4 \\
\hline Lauwers [54] & 1 & 0 & 1 & 0 & 2 & 1 & 0 & 0 & 5 \\
\hline Controni [55] & 1 & 0 & 1 & 0 & 1 & 1 & 0 & 1 & 5 \\
\hline Bland [56] & 0 & 0 & 0 & 0 & 2 & 2 & 0 & 1 & 5 \\
\hline
\end{tabular}

${ }^{\mathrm{a} S t u d y}$ design (cross-sectional or case-control); ${ }^{\mathrm{b}}$ Data collection (prospective or retrospective); ${ }^{c}$ recruitment of the patient (consecutive or random); ${ }^{\mathrm{d}}$ exclusion criteria; ${ }^{\text {p }}$ prior antibiotic treatment; ${ }^{\mathrm{f}}$ tuberculous meningitis; ${ }^{\mathrm{g}}$ blinded interpretation of lactate measurement; ${ }^{\mathrm{h}}$ reliability of the method (stabilizer for lactate sample or immediate measurement).

sensitivity, LR-, and DOR were significantly homogenous (data not shown). Symmetrical SROC fitting was also performed for these five studies due to a constant DOR (testing whether parameter $b=0, P=0.4452$ ). The result showed a SROC curve with the $Q$ value and AUC at 0.9426 and 0.9828 , respectively, indicating excellent accuracy, and was consistent with the 25 included studies (data not shown).

\section{Sub meta-analysis of lactate as a differential marker for diagnosed BM from diagnosed viral AM}

Meta-analysis was further performed to assess the diagnostic accuracy of lactate between diagnosed BM and diagnosed viral AM. One study that recruited only diagnosed viral $A M$ and four other studies that included diagnosed viral AM as well as clinical AM that could be extracted separately were included in the subgroup analysis. The specificity was still heterogeneous among the studies $\left(\chi^{2} P=0.14, I^{2}=42.1 \%\right)$ of diagnostic accuracy, but sensitivity, LR+, LR-, and DOR were significantly homogenous (data not shown). Symmetrical SROC fitting was also performed for these five studies due to a constant DOR (testing whether parameter $b=0$,
$P=0.9145)$. The result revealed a SROC curve with the $\mathrm{Q}$ value and AUC at 0.9563 and 0.9891 , respectively, suggesting excellent accuracy, and was consistent with above results (data not shown).

\section{Head-to-head comparison of CSF lactate level versus conventional markers}

In order to compare the diagnostic accuracy of the CSF lactate concentration and other conventional markers for diagnosis of BM, data were extracted from the 25 selected articles only if the study had on the same set of specimens a parallel analysis of CSF lactate and a conventional marker. Since conventional markers were used as the diagnostic criteria of BM, only BM patients with confirmed diagnosis were extracted in this analysis. The extracted data are shown in Table 3, which includes the DOR values for CSF lactate, CSF glucose, CSF/plasma glucose quotient, CSF protein, CSF total number leukocytes, CSF percentages of granulocytes, and CSF number of granulocytes.

In the present study, for diagnosis of BM, five studies performed head to head comparisons of CSF lactate versus CSF glucose, four versus the CSF/plasma glucose 
A

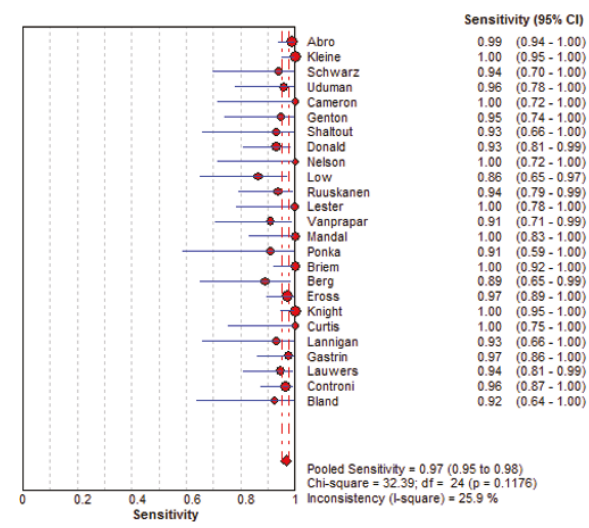

C

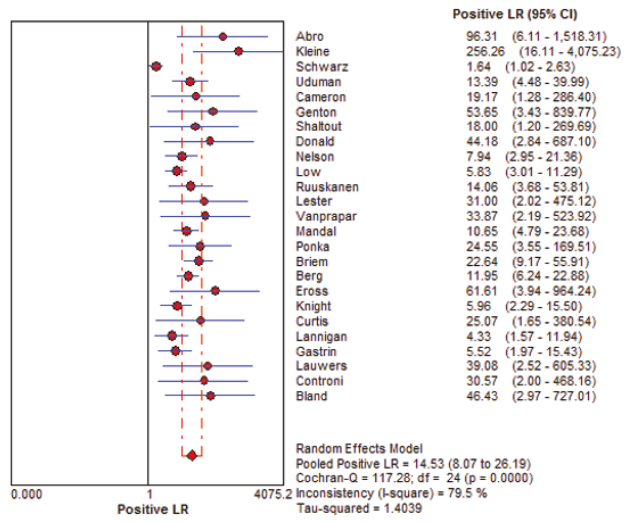

E

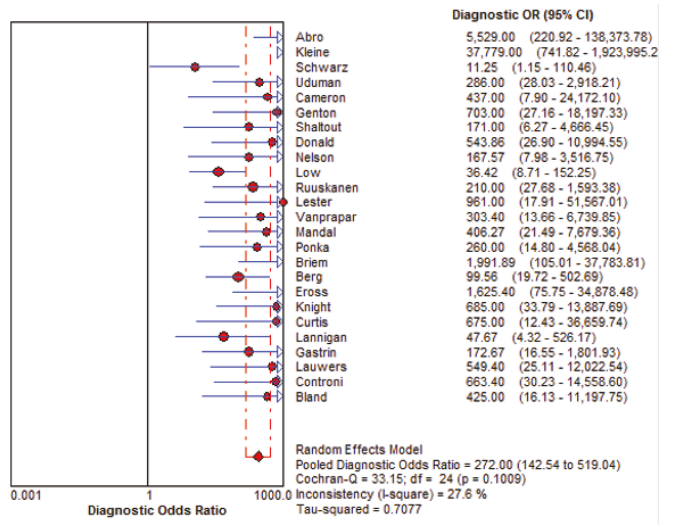

Analysis Options:
Add 12 to all cells of the studies with zero
Fifter OFF
B

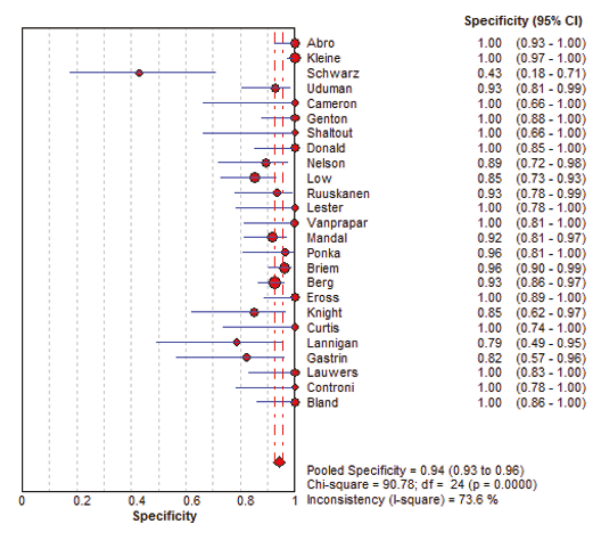

D

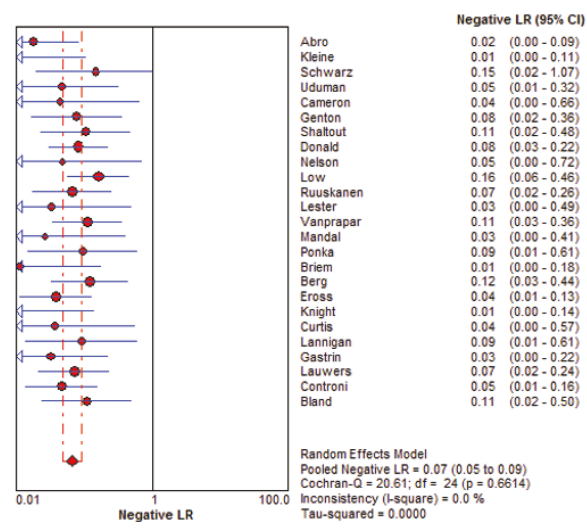

Figure 2 Diagnostic accuracy of the CSF lactate concentration for differential diagnosis of BM from AM. Forest plot showing sensitivity, specificity, LR+, LR-, and DOR with $95 \%$ confidence intervals $(95 \% \mathrm{Cl})$ for the lactate concentration for differential diagnosis of BM from AM. The size of the circle represents the study size.

quotient, seven versus CSF protein, five versus CSF total number of leukocytes, one versus percentages of granulocytes, and one versus CSF number of granulocytes. However, TB meningitis patients and partially treated BM patients could not be excluded from the conventional markers assays. Therefore, in a secondary meta-analysis these patients were included in the BM group. Higher DOR values were observed with the CSF lactate level than with the conventional markers in all studies except for one study for the CSF protein assay [40] and one 


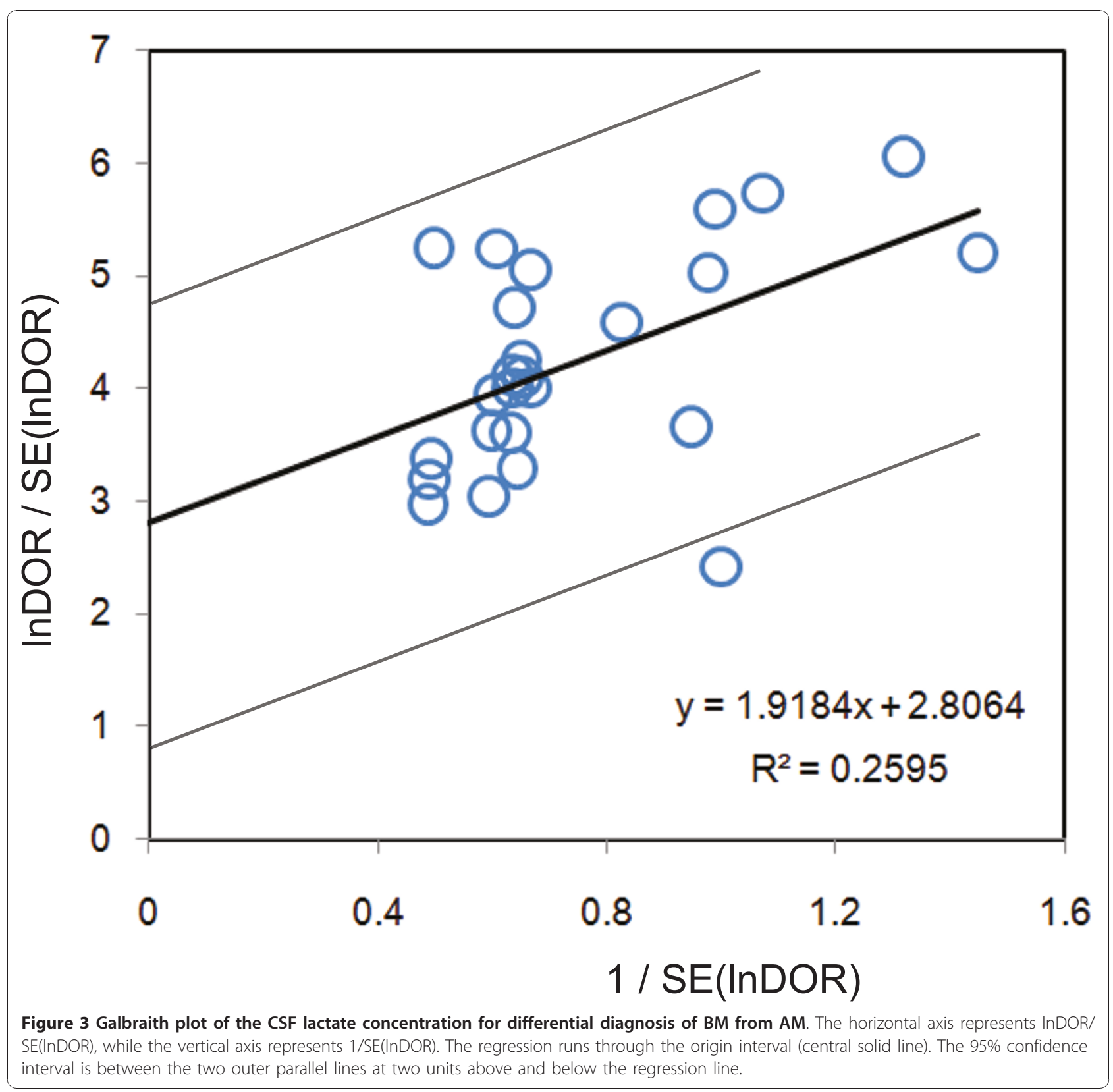

study for total number of leukocytes [42]. Since DOR values of the CSF lactate concentration, CSF glucose level, CSF/plasma glucose quotient, and CSF total number of leukocytes were found to be constant (data not shown), symmetrical SROC fitting by a random effects model was performed for these assays. On the other hand, asymmetrical SROC fitting by a random effects model was computed for the CSF protein assay because the slope of the fitted regression line of the Moses-Shapiro-Littenberg model was not zero (data not shown). Following SROC analysis for all four subgroups of the CSF lactate concentration (Figure 5), the overall AUC was 0.977 to 0.988 , which was consistent with the primary analysis of the 25 included studies. In addition, the AUC values were found to be lower for the four conventional markers $(0.881,0.952,0.862$, and 0.948 for CSF glucose, CSF/plasma glucose quotient, CSF protein, and CSF total number of leukocytes, respectively), suggesting a lower accuracy compared to the CSF lactate test.

\section{Assessment of publication bias}

The relatively asymmetric funnel plot (Figure 6) and the Egger intercept $(2.95$, two-tailed $P=0.00004)$ suggested the presence of a publication bias. Using the trim and fill method of Duvall and Tweedie, 11 missing studies were required in the left side of the funnel plot in order 


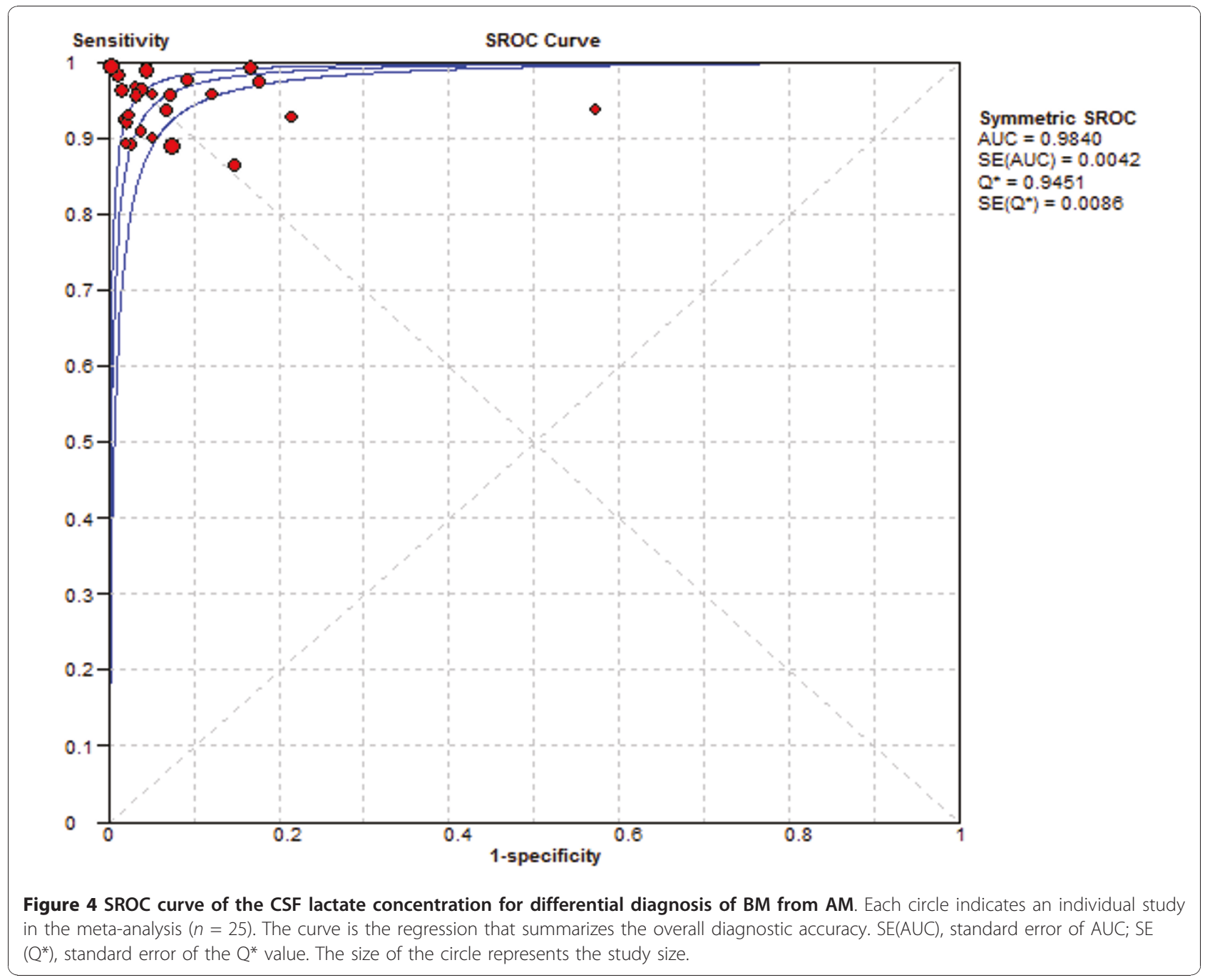

to make the plot symmetric. However, the pooled lnDOR dropped just slightly from 5.60 (95\% CI, 4.95 to $.25)$ to 4.84 (95\% CI, 4.16 to 5.53 ) after addition of these missing studies.

\section{Discussion}

The present meta-analysis revealed that the AUC of CSF lactate concentration was 0.9840 (Figure 4), indicating an excellent level of overall accuracy. The overall performance was highest for the CSF lactate concentration compared to the performances of the four conventional markers (CSF glucose, CSF/plasma glucose quotient, CSF protein, and CSF total number of leukocytes) based on head-to-head meta-analytic SROC curves and their AUC (Figure 5), which was in good agreement with previous literature $[4,59]$. CSF lactate is less useful if it has a low concentration, but the assay is supportive if it is positive, especially if the diagnosis was otherwise not conclusive. In such cases, increased CSF lactate should be considered a sign of BM. Because of the lactate assay, several BM patients with elevated CSF lactate and minimal CSF abnormalities have been treated with antibiotics prior to culture test results $[11,47,55]$. Moreover, an increased CSF lactate level has been also proposed as a good indicator of CSF infection in intra-ventricular hemorrhagic patients with an external ventricular drain $[60,61]$. However, clinicians should be aware that CSF lactate is also increased in several central nervous system diseases such stroke (2 to $8 \mathrm{mmol} / \mathrm{l}$ ) [62,63], convulsion ( 2 to $4 \mathrm{mmol} / \mathrm{l}$ ) [64], cerebral trauma (2 to $9 \mathrm{mmol} / \mathrm{l}$ ) [52], hypoglycemic coma (2 to $6 \mathrm{mmol} / \mathrm{l}$ ) [65].

The measurement of CSF lactate concentration is a simple, rapid, inexpensive assay, takes just 15 minutes, and can be performed at the bedside. In addition, the CSF lactate concentration is useful during the course of treatment, because a rapid CSF lactate decrease is indicative of good prognosis [39]. Since the CSF lactate concentration is not specific for BM, the results of this 
Table 3 Head-to-head comparison of CSF lactate concentration and other conventional markers

\begin{tabular}{|c|c|c|c|c|c|c|c|c|c|c|c|}
\hline \multirow[t]{2}{*}{ Study (ref) } & \multirow[t]{2}{*}{ Conventional markers (location) } & \multicolumn{5}{|c|}{ Conventional markers assay results } & \multicolumn{5}{|c|}{ Lactate assay results } \\
\hline & & TP & FP & FN & TN & DOR & TP & FP & FN & TN & DOR \\
\hline Shaltout ${ }^{\mathrm{a}, \mathrm{b}, \mathrm{c}}[40]$ & Glucose (CSF) & 10 & 1 & 6 & 41 & 68.3 & 14 & 1 & 2 & 41 & 287.0 \\
\hline Donald $^{\mathrm{a}, \mathrm{c}}[41]$ & & 33 & 2 & 15 & 67 & 73.7 & 45 & 3 & 3 & 69 & 345.0 \\
\hline Pönkä [47] & & 5 & 0 & 5 & 27 & 55.0 & 10 & 1 & 1 & 26 & 260.0 \\
\hline Briem $^{\mathrm{a}, \mathrm{c}}[48]$ & & 30 & 0 & 23 & 193 & 502.3 & 47 & 4 & 0 & 167 & $3,536.1$ \\
\hline Lannigan [53] & & 11 & 4 & 2 & 10 & 13.8 & 13 & 3 & 1 & 11 & 47.7 \\
\hline Genton $^{a, b}[39]$ & Glucose quotient (CSF/plasma) & 21 & 0 & 2 & 27 & 473.0 & 24 & 0 & 1 & 28 & 931.0 \\
\hline Nelson ${ }^{\mathbf{b}}[42]$ & & 10 & 0 & 7 & 26 & 74.2 & 18 & 3 & 0 & 25 & 269.6 \\
\hline Briem $^{\mathrm{a}, \mathrm{c}}[48]$ & & 40 & 1 & 13 & 191 & 587.7 & 47 & 4 & 0 & 167 & $3,536.1$ \\
\hline Berg $^{a}[49]$ & & 16 & 10 & 2 & 78 & 62.4 & 16 & 9 & 2 & 112 & 99.6 \\
\hline Genton $^{a, b}[39]$ & Protein concentration (CSF) & 18 & 0 & 3 & 25 & 269.6 & 24 & 0 & 1 & 28 & 931.0 \\
\hline Shaltout $\mathrm{t}^{\mathrm{a}, \mathrm{b}, \mathrm{c}}[40]$ & & 13 & 0 & 3 & 42 & 327.9 & 14 & 1 & 2 & 41 & 287.0 \\
\hline Donald $^{\mathrm{a}, \mathrm{c}}$ & & 39 & 1 & 9 & 68 & 294.7 & 45 & 3 & 3 & 69 & 345.0 \\
\hline Vanprapar [45] & & 8 & 0 & 3 & 12 & 60.7 & 12 & 0 & 1 & 18 & 308.3 \\
\hline Pönkä [47] & & 10 & 11 & 1 & 16 & 14.5 & 10 & 1 & 1 & 26 & 260.0 \\
\hline Briem $^{\mathrm{a}, \mathrm{c}}$ [48] & & 41 & 7 & 9 & 184 & 119.8 & 47 & 4 & 0 & 167 & $3,536.1$ \\
\hline Berg $^{\mathbf{a}}[49]$ & & 11 & 14 & 4 & 88 & 17.3 & 16 & 9 & 2 & 112 & 99.6 \\
\hline Genton $^{\mathbf{a}, \mathbf{b}}[39]$ & Leukocytes (CSF total number) & 16 & 0 & 8 & 26 & 102.9 & 24 & 0 & 1 & 28 & 931.0 \\
\hline Shaltout ${ }^{a, b, c}[40]$ & & 10 & 2 & 6 & 39 & 32.5 & 14 & 1 & 2 & 41 & 287.0 \\
\hline Nelson $^{\mathbf{b}}[42]$ & & 17 & 1 & 1 & 26 & 442.0 & 18 & 3 & 0 & 25 & 269.6 \\
\hline Pönkä [47] & & 7 & 2 & 4 & 25 & 21.9 & 10 & 1 & 1 & 26 & 260.0 \\
\hline Lannigan [53] & & 12 & 2 & 2 & 12 & 36.0 & 13 & 3 & 1 & 11 & 47.7 \\
\hline Genton $^{a, b}[39]$ & Granulocytes (CSF \%) & 20 & 7 & 4 & 19 & 13.6 & 24 & 0 & 1 & 28 & 931.0 \\
\hline Pönkä [47] & Neutrophils (CSF number) & 7 & 6 & 4 & 21 & 6.1 & 10 & 1 & 1 & 26 & 260.0 \\
\hline
\end{tabular}

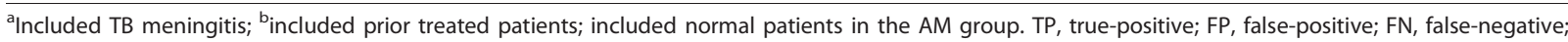

$\mathrm{TN}$, true-negative; DOR, diagnostic odds ratio.

assay should be interpreted in parallel with clinical findings and the results of conventional assays including CSF concentrations of protein, cells, glucose, and a microbiological examination of CSF. The cut-off value for CSF lactate concentration ranges from 2.1 to $4.44 \mathrm{mmol} / \mathrm{L}$, suggesting a variance between instrument, hospital labs, and the method. Therefore, every center should set its own cut-off value for CSF lactate concentration. Another disadvantage of CSF lactate is that it is not useful in the choice of antibiotic selection, which must be based on the results of microscopic examination of a smear or culture for bacteria, as well as the other clinical data.

The mechanism of the increased concentration of lactate in the CSF of patients with BM is not clear, but it has been linked with anaerobic glycolysis of brain tissue due to a decrease cerebral blood flow and oxygen uptake [66,67]. Additionally, the concentration of CSF lactate is independent of serum lactate, probably due to its ionized state that crosses the blood-CSF barrier at a very slow rate [68], suggesting another advantage over CSF glucose assay [38].

The present systemic review has several strengths. First, the criteria and protocol were defined, the protocol was followed, and a search of several databases and sources was performed to identify potential studies. The quality of included studies was assessed by using several criteria that could affect diagnostic accuracy. These steps were carried out by two independent researchers. Heterogeneity was explored in accordance with published guidelines. Then, the summary ROC curve was computed and Q values and AUC were calculated in order to evaluate the diagnostic accuracy of CSF lactate marker. Potential effects of several covariates on the diagnostic accuracy were assessed, but none were found.

Because publication bias can affect the accuracy of diagnostic assays, potential publication bias was assessed using funnel plots. The results showed a skewed funnel shape, suggesting a potential publication bias in the literature (Figure 6). However, it was noted that the three largest studies $[37,48,50]$ had higher DORs compared to smaller studies, and they had similar almost perfect accuracy $[38,44,56]$. This discrepancy could be explained by the calculation method of adding 0.5 to cells with zero, suggesting a weakness of the funnel plot when the assay investigated has excellent accuracy. Another main concern is the lack of some additional databases that 


\section{A}

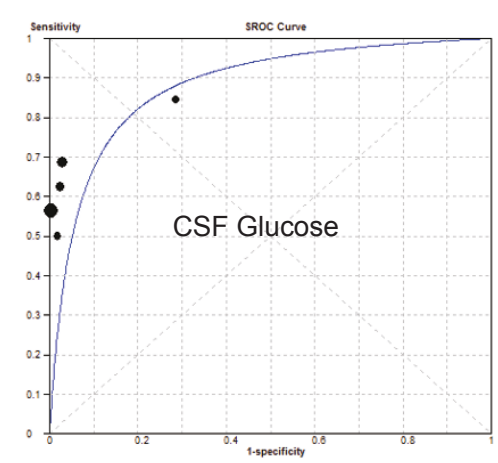

B

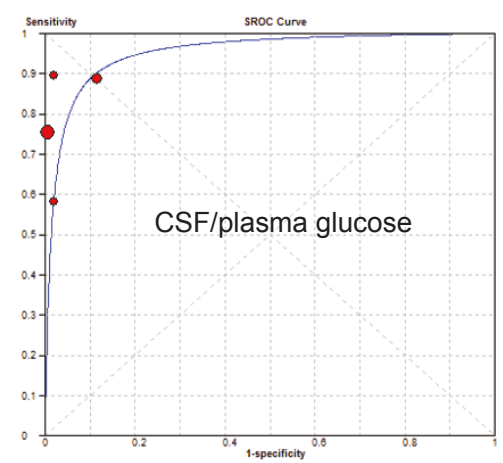

C

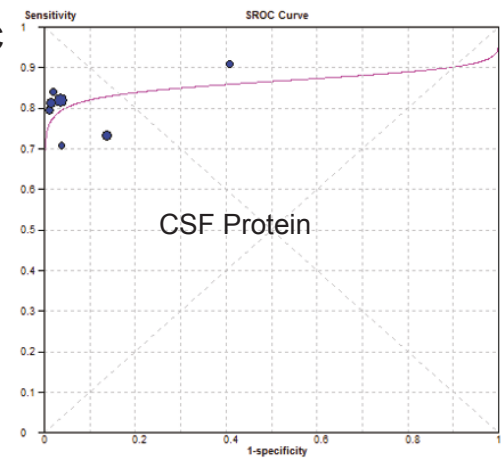

D

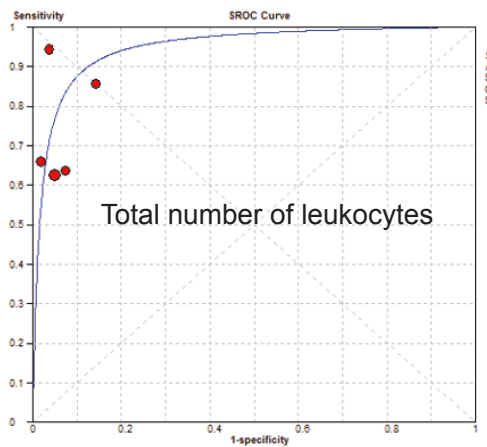

sroc curve
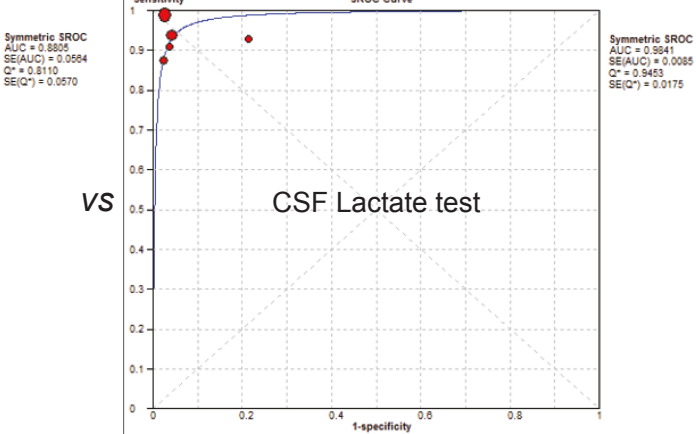

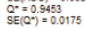
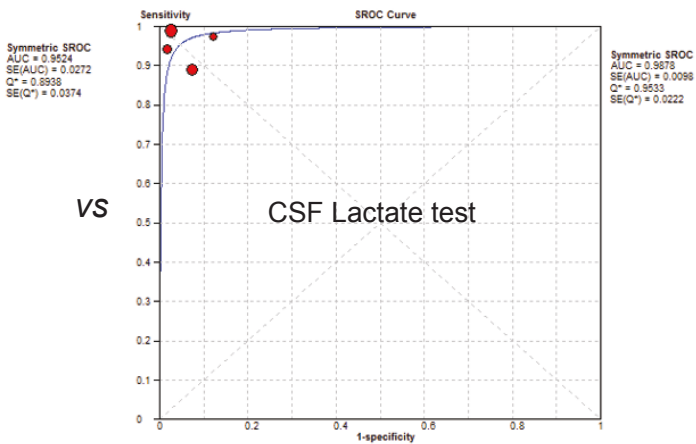

${ }_{\mathrm{SE}\left(0^{\circ}\right)=0.0222}$

vs
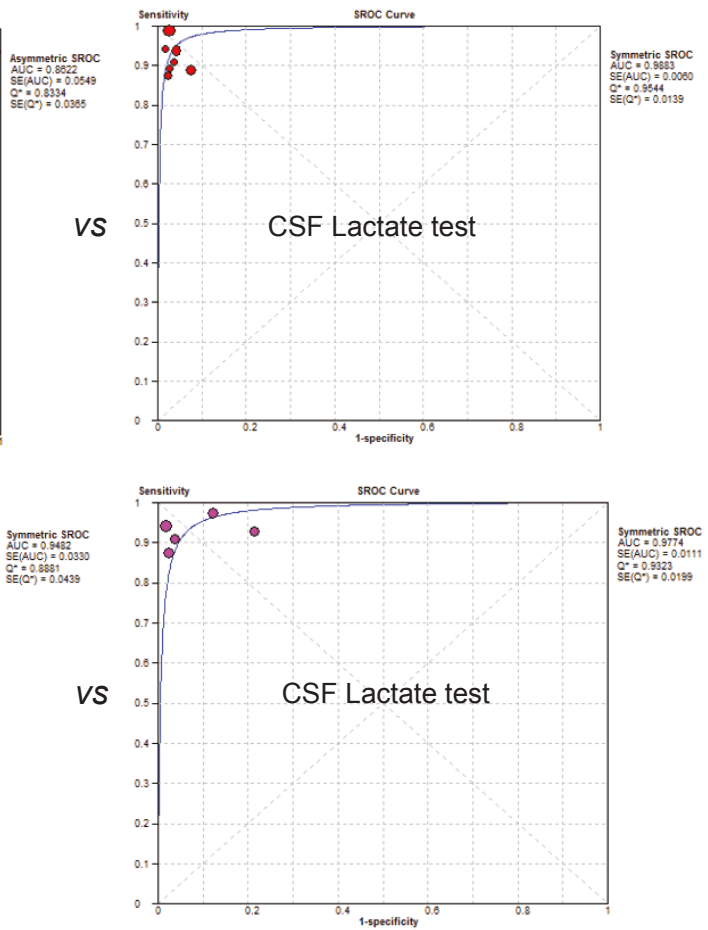

Figure 5 SROC curve of the head to head comparison of the CSF lactate concentration and other conventional markers. Each circle indicates an individual study in the meta-analysis. The curve is the regression that summarizes the overall diagnostic accuracy. SE(AUC), standard error of $A \cup C ; \operatorname{SE}\left(Q^{*}\right)$, standard error of $Q^{*}$ value. The size of the circle represents the study size. 


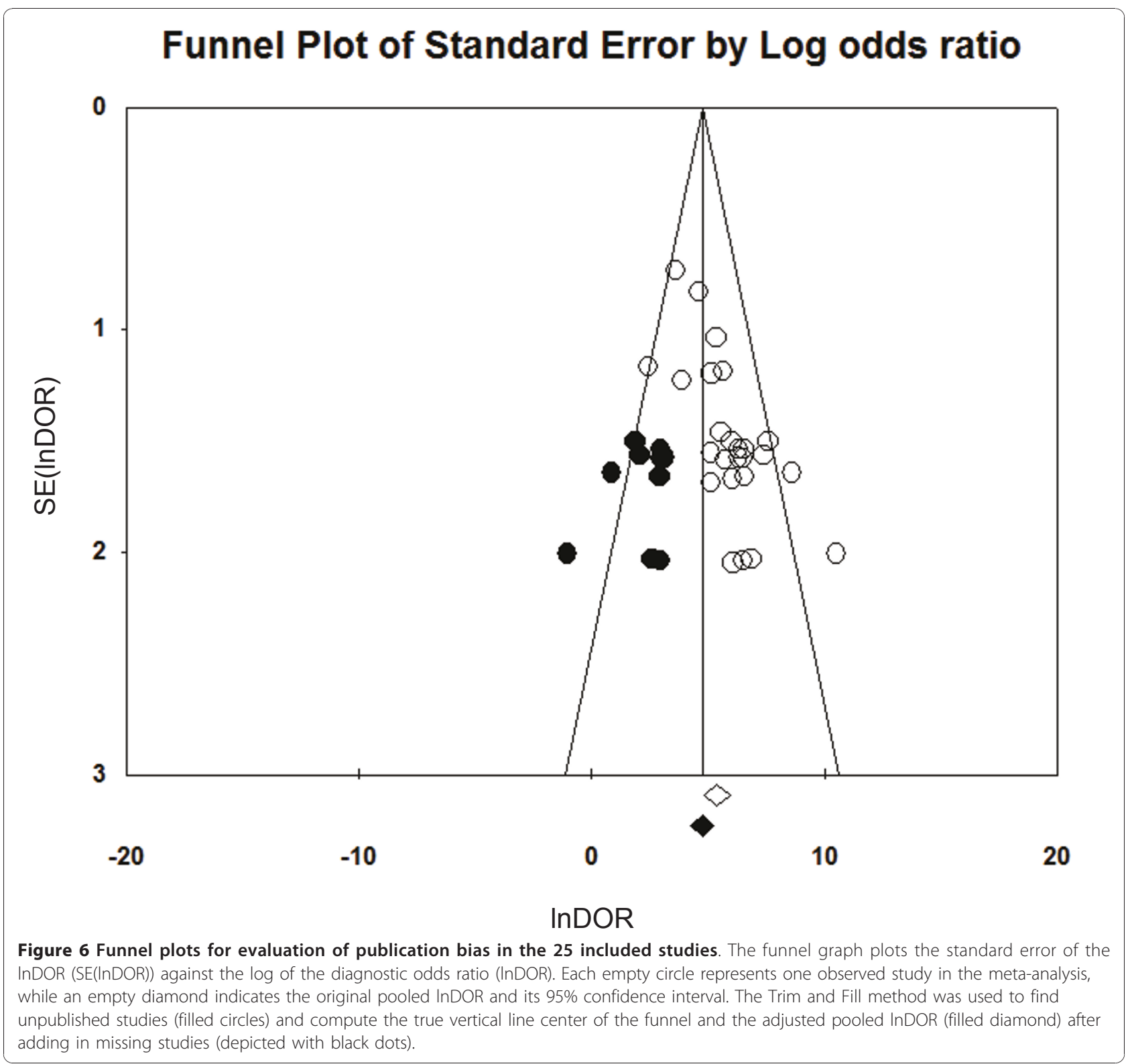

were used for searching, that is, we did not access EMBASE, which could have added more relevant studies. we did search Scopus, which is reportedly $91.6 \%$ overlapped with EMBASE [69]. Therefore, we think that we have not missed many studies large enough to change the overall impression of our results.

In addition, non-English language studies were also excluded; the non-English language reports represented approximately $10 \%$ of all initial articles. We excluded non-English articles in meta-analyses due to limited resource and potential error in the translation and interpretation in several languages including Chinese, Croatian, Dutch, French, German, Hebrew, Italian, Korean, Norwegian, Polish, Portuguese, Romanian, Russian,
Serbian, Spanish, and Turkish. The odds ratio in metaanalyses from non-English articles is reportedly 0.8 (95\% CI, 0.7 to 1.0) times lower than that from Englishwritten publications [70], therefore, it is unlikely that the inclusion of these non-English articles would have altered our main conclusions substantially.

In addition, studies that reported non-significant results are less likely to be accepted for publication. All of these potentially missing data could result in a significant publication bias. However, the trim and fill method of Duvall and Tweedie was used to overcome this bias, and it was found that it was unlikely to distort the overall diagnostic performance of the lactate concentration (Figure 6). 


\section{Conclusions}

The present meta-analysis study indicated that for discrimination of BM from AM, the CSF lactate concentration is a good single indicator and a better marker compared to other conventional markers including CSF glucose, CSF/plasma glucose quotient, CSF protein, and CSF total number of leukocytes. Cost-effectiveness studies should be performed to investigate the economic impact of using this technique as a routine assay in hospital to distinguish BM from AM.

\section{Key messages}

- The diagnostic accuracy of cerebrospinal fluid (CSF) lactate assay for differential diagnosis between bacterial meningitis and aseptic meningitis was excellent with Q value of 0.9451 and area under the curve of 0.9840 .

- CSF lactate was a better marker for distinguishing bacterial meningitis from aseptic meningitis compared to other conventional markers including CSF glucose, CSF/plasma glucose quotient, CSF protein, and CSF total number of leukocytes

\begin{abstract}
Abbreviations
AM: aseptic meningitis; AUC: area under the curve; BM: bacterial meningitis; Cl: confidence interval; CRP: C-reactive protein; CSF: cerebrospinal fluids; DOR: Diagnostic odd ratio; ESS: effective sample size; InDOR: natural log of DOR, LR+, positive likelihood ratio; LR-: negative likelihood ratio, ROC, receiver-operator characteristic; SE: standard error; SROC: summary ROC curve; TB: tuberculosis.
\end{abstract}

\section{Acknowledgements}

This work was supported in part by a Grant-in-Aid for Young Scientists (17301870, 2008-2010 for NTH) from the Ministry of Education, Culture, Sports, Science and Technology (MEXT, Japan), and was supported in part by a Grant-in-Aid for Scientific Research from Nagasaki University to NTH (2007-2009). This study was also supported in part by the Global COE Program (2008-2012) and Japan Initiative for Global Research Network on Infectious Diseases (J-GRID) for KH.

\section{Author details}

${ }^{1}$ Department of Immunogenetics, Institute of Tropical Medicine (NEKKEN), Nagasaki University, 1-12-4 Sakamoto, Nagasaki 852-8523, Japan. ${ }^{2}$ Department of Pediatrics, University of Medicine and Pharmacy at Ho Chi Minh City, 217 Hong Bang Street, District 5, Ho Chi Minh City, Vietnam. ${ }^{3}$ Children's Hospital No.1, 2 Su Van Hanh Street, District 10, Ho Chi Minh City, Vietnam. ${ }^{4}$ Center for International Collaborative Research, Nagasaki University, 1-12-4 Sakamoto, Nagasaki 852-8523, Japan. ${ }^{5}$ Clinical Biostatistics Unit, Ramón y Cajal Hospital, Carretera de Colmenar km 9.100, 28034 Madrid, Spain. ${ }^{6}$ Global COE program, Nagasaki University, 1-12-4 Sakamoto, Nagasaki 852-8523, Japan.

\section{Authors' contributions}

NTH designed the research, collected, analyzed and interpreted the data, and drafted and revised the manuscript. NTHT carried out the collection, analysis and interpretation of the data. DTND and MK contributed to the conception of the study and approved the final version of the manuscript. JZ helped to design the study, performed the statistical analysis and drafted the manuscript. $\mathrm{KH}$ participated in the design of the study and drafted and revised the manuscript. All authors read and approved the final manuscript.

\section{Competing interests}

The authors declare that they have no competing interests.
Received: 17 June 2010 Revised: 21 September 2010

Accepted: 31 December 2010 Published: 31 December 2010

\section{References}

1. Saez-Llorens $X$, McCracken GH Jr: Bacterial meningitis in children. Lancet 2003, 361:2139-2148

2. Durand ML, Calderwood SB, Weber DJ, Miller SI, Southwick FS, Caviness VS $\mathrm{Jr}$, Swartz MN: Acute bacterial meningitis in adults. A review of 493 episodes. N Engl J Med 1993, 328:21-28.

3. Shelburne C, Statler M: Meningitis: distinguishing the benign from the serious. Jaapa 2008, 21:54-59.

4. Roos KL, Tyler KL: Meningitis, Encephalitis, Brain abscess, and Empyema. Harrison's Principles of Internal Medicine. 16th edition. New York: McGraw-Hill Professional; 2004, 2471-2490

5. Jacques J, Carquin J, Brodard V, Moret H, Lebrun D, Bouscambert M, Motte J, Remy G, Andreoletti L: New reverse transcription-PCR assay for rapid and sensitive detection of enterovirus genomes in cerebrospinal fluid specimens of patients with aseptic meningitis. J Clin Microbiol 2003, 41:5726-5728

6. Freeman HR, Mai NT, Diep TS, Parry C, Hien TT, Farrar JJ: The role of the polymerase chain reaction in the diagnosis of bacterial meningitis in Vietnam. Ann Trop Med Parasitol 2004, 98:65-70.

7. Wise R, Hart T, Cars O, Streulens M, Helmuth R, Huovinen P, Sprenger M: Antimicrobial resistance. Is a major threat to public health. BMJ 1998, 317:609-610

8. Raymond J: [Epidemiology of nosocomial infections in pediatrics]. Pathol Biol (Paris) 2000, 48:879-884.

9. Parasuraman TV, Frenia K, Romero J: Enteroviral meningitis. Cost of illness and considerations for the economic evaluation of potential therapies. Pharmacoeconomics 2001, 19:3-12.

10. Cunha BA: Distinguishing bacterial from viral meningitis: the critical importance of the CSF lactic acid levels. Intensive Care Med 2006, 32:1272-1273, author reply 1274

11. Gastrin B, Briem H, Rombo L: Rapid diagnosis of meningitis with use of selected clinical data and gas-liquid chromatographic determination of lactate concentration in cerebrospinal fluid. J Infect Dis 1979, 139:529-533.

12. Ruuskanen O, Stahlberg ML, Korvenranta H, Nikoskelainen J, Irjala K: CSF lactate in bacterial meningitis with minimal CSF abnormalities. Acta Paediatr Scand 1985, 74:292-293.

13. Rajs G, Finzi-Yeheskel Z, Rajs A, Mayer M: C-reactive protein concentrations in cerebral spinal fluid in gram-positive and gram-negative bacterial meningitis. Clin Chem 2002, 48:591-592.

14. Viallon A, Pouzet V, Zeni F, Tardy B, Guyomarc'h S, Lambert C, Page Y, Bertrand JC: [Rapid diagnosis of the type of meningitis (bacterial or viral) by the assay of serum procalcitonin]. Presse Med 2000, 29:584-588.

15. Moher D, Cook DJ, Eastwood S, Olkin I, Rennie D, Stroup DF: Improving the quality of reports of meta-analyses of randomised controlled trials: the QUOROM statement. Quality of Reporting of Meta-analyses. Lancet 1999, 354:1896-1900.

16. The PRISMA Statement. [http://www.plosmedicine.org/article/info\%3Adoi\% 2F10.1371\%2Fjournal.pmed.1000100].

17. The PubMed Database. [http://www.ncbi.nlm.nih.gov/sites/entrez].

18. Scopus. [http://www.scopus.com/home.url].

19. MEDION database. [http://www.mediondatabase.nl]

20. Cochrane Library. [http://www.cochrane.org].

21. Chavanet P, Schaller C, Levy C, Flores-Cordero J, Arens M, Piroth L

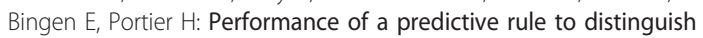
bacterial and viral meningitis. J Infect 2007, 54:328-336.

22. Deville $W L$, Buntinx F, Bouter LM, Montori VM, de Vet HC, van der Windt DA, Bezemer PD: Conducting systematic reviews of diagnostic studies: didactic guidelines. BMC Med Res Methodol 2002, 2:9.

23. Pai M, McCulloch M, Enanoria W, Colford JM Jr: Systematic reviews of diagnostic test evaluations: What's behind the scenes? ACP J Club 2004, 141:A11-13.

24. Lijmer JG, Mol BW, Heisterkamp S, Bonsel GJ, Prins MH, van der Meulen JH, Bossuyt PM: Empirical evidence of design-related bias in studies of diagnostic tests. JAMA 1999, 282:1061-1066

25. Brook I: Stability of lactic acid in cerebrospinal fluid specimens. Am J Clin Pathol 1982, 77:213-216. 
26. Zamora J, Abraira V, Muriel A, Khan K, Coomarasamy A: Meta-DiSc: a software for meta-analysis of test accuracy data. BMC Med Res Methodol 2006, 6:31.

27. Meta-Disc (version 1.4) software. [http://www.hrc.es/investigacion/ metadisc_en.htm]

28. Moses LE, Shapiro D, Littenberg B: Combining independent studies of a diagnostic test into a summary ROC curve: data-analytic approaches and some additional considerations. Stat Med 1993, 12:1293-1316.

29. Higgins JP, Thompson SG, Deeks JJ, Altman DG: Measuring inconsistency in meta-analyses. BMJ 2003, 327:557-560.

30. Galbraith RF: A note on graphical presentation of estimated odds ratios from several clinical trials. Stat Med 1988, 7:889-894.

31. Deeks JJ, Macaskill P, Irwig L: The performance of tests of publication bias and other sample size effects in systematic reviews of diagnostic test accuracy was assessed. J Clin Epidemiol 2005, 58:882-893.

32. Jones CM, Athanasiou T: Summary receiver operating characteristic curve analysis techniques in the evaluation of diagnostic tests. Ann Thorac Surg 2005, 79:16-20.

33. Egger M, Davey Smith G, Schneider M, Minder C: Bias in meta-analysis detected by a simple, graphical test. BMJ 1997, 315:629-634.

34. Duval S, Tweedie R: Trim and fill: A simple funnel-plot-based method of testing and adjusting for publication bias in meta-analysis. Biometrics 2000, 56:455-463.

35. Mengoli C, Cruciani M, Barnes RA, Loeffler J, Donnelly JP: Use of PCR for diagnosis of invasive aspergillosis: systematic review and meta-analysis. Lancet Infect Dis 2009, 9:89-96.

36. The Comprehensive Meta-analysis software version 2.0. [http://www.meta-analysis.com].

37. Abro AH, Abdou AS, Ali H, Ustadi AM, Hasab AAH: Cerebrospinal fluid analysis acute bacterial versus viral meningitis. Pak J Med Sci 2008, 24:645-650 [http://pjms.com.pk/issues/octdec108/article/article1.html].

38. Cameron PD, Boyce JM, Ansari BM: Cerebrospinal fluid lactate in meningitis and meningococcaemia. J Infect 1993, 26:245-252.

39. Genton B, Berger JP: Cerebrospinal fluid lactate in 78 cases of adult meningitis. Intensive Care Med 1990, 16:196-200.

40. Shaltout AA, Helal AA, Awadallah NB, Mughal HA, Johny M: Cerebrospinal fluid lactate is useful in differentiating viral from bacterial meningitis. Medical Principles and Practice 1989, 1:12-16 [http://content.karger.com/ ProdukteDB/produkte.asp?doi=157274].

41. Donald PR, Malan C: Cerebrospinal fluid lactate and lactate dehydrogenase activity in the rapid diagnosis of bacterial meningitis. $S$ Afr Med J 1986, 69:39-42.

42. Nelson N, Eeg-Olofsson O, Larsson L, Ohman S: The diagnostic and predictive value of cerebrospinal fluid lactate in children with meningitis. Its relation to current diagnostic methods. Acta Paediatr Scand 1986, 75:52-57.

43. Low PS, Yip WC, Tay JS, Wong HB: Cerebrospinal fluid lactate-its diagnostic value in septic meningitis. J Singapore Paediatr Soc 1986, 28:243-246.

44. Lester A, Stilbo I, Bartels P, Bruun B: Value of CSF lactate in the differential diagnosis between bacterial meningitis and other diseases with meningeal involvement. Acta Pathol Microbiol Immunol Scand ([B) 1985, 93:21-25.

45. Vanprapar N, Chavalittamrong B, Limsathayurat N: Cerebrospinal fluid lactate level in childhood purulent meningitis. Southeast Asian J Trop Med Public Health 1983, 14:186-190.

46. Mandal BK, Dunbar EM, Hooper J, Parker L: How useful is cerebrospinal fluid lactate estimation in differential diagnosis of meningitis? J Infect 1983, 6:231-237.

47. Ponka A, Ojala K, Teppo AM, Weber TH: The differential diagnosis of bacterial and aseptic meningitis using cerebrospinal fluid laboratory tests. Infection 1983, 11:129-131.

48. Briem H: Comparison between cerebrospinal fluid concentrations of glucose, total protein, chloride, lactate, and total amino acids for the differential diagnosis of patients with meningitis. Scand J Infect Dis 1983, 15:277-284.

49. Berg B, Gardsell P, Skansberg P: Cerebrospinal fluid lactate in the diagnosis of meningitis. Diagnostic value compared to standard biochemical methods. Scand J Infect Dis 1982, 14:111-115.

50. Eross J, Silink M, Dorman D: Cerebrospinal fluid lactic acidosis in bacterial meningitis. Arch Dis Child 1981, 56:692-698.
51. Knight JA, Dudek SM, Haymond RE: Early (chemical) diagnosis of bacterial meningitis-cerebrospinal fluid glucose, lactate, and lactate dehydrogenase compared. Clin Chem 1981, 27:1431-1434.

52. Curtis GD, Slack MP, Tompkins DS: Cerebrospinal fluid lactate and the diagnosis of meningitis. J Infect 1981, 3:159-165.

53. Lannigan R, MacDonald MA, Marrie TJ, Haldane EV: Evaluation of cerebrospinal fluid lactic acid levels as an aid in differential diagnosis of bacterial and viral meningitis in adults. J Clin Microbiol 1980, 11:324-327.

54. Lauwers S: Lactic-acid concentration in cerebrospinal fluid and differential diagnosis of meningitis. Lancet 1978, 2:163.

55. Controni G, Rodriguez WJ, Hicks JM, Ficke M, Ross S, Friedman G, Khan W: Cerebrospinal fluid lactic acid levels in meningitis. J Pediatr 1977, 91:379-384.

56. Bland RD, Lister RC, Ries JP: Cerebrospinal fluid lactic acid level and pH in meningitis. Aids in differential diagnosis. Am J Dis Child 1974, 128:151-156.

57. Uduman SA, Adeyemi E, El-Khadir A, Jose K, Benedict S, Bener A: Haemophilus influenzae type $B$ still remains a leading cause of meningitis among unvaccinated children-a prospective CSF analysis study. J Trop Pediatr 2000, 46:331-334.

58. Schwarz S, Bertram M, Schwab S, Andrassy K, Hacke W: Serum procalcitonin levels in bacterial and abacterial meningitis. Crit Care Med 2000, 28:1828-1832.

59. Kleine TO, Zwerenz P, Zofel P, Shiratori K: New and old diagnostic markers of meningitis in cerebrospinal fluid (CSF). Brain Res Bull 2003, 61:287-297.

60. Wong GK, Poon WS, Ip M: Use of ventricular cerebrospinal fluid lactate measurement to diagnose cerebrospinal fluid infection in patients with intraventricular haemorrhage. J Clin Neurosci 2008, 15:654-655.

61. Sommer JB, Gaul C, Heckmann J, Neundorfer B, Erbguth FJ: Does lumbar cerebrospinal fluid reflect ventricular cerebrospinal fluid? A prospective study in patients with external ventricular drainage. Eur Neurol 2002, 47:224-232.

62. Busse $\mathrm{O}$, Hoffmann $\mathrm{O}$ : CSF lactate and $\mathrm{CT}$ findings in middle cerebral artery infarction. A comparative study. Stroke 1983, 14:960-963.

63. Fujishima M, Sugi T, Choki J, Yamaguchi T, Omae T: Cerebrospinal fluid and arterial lactate, pyruvate and acid-base balance in patients with intracranial hemorrhages. Stroke 1975, 6:707-714.

64. Simpson $\mathrm{H}$, Habel AH, George EL: Cerebrospinal fluid acid-base status and lactate and pyruvate concentrations after convulsions of varied duration and aetiology in children. Arch Dis Child 1977, 52:844-849.

65. Yao H, Sadoshima S, Nishimura Y, Fujii K, Oshima M, Ishitsuka T, Fujishima M: Cerebrospinal fluid lactate in patients with diabetes mellitus and hypoglycaemic coma. J Neurol Neurosurg Psychiatry 1989, 52:372-375.

66. Menkes $\mathrm{JH}$ : The causes for low spinal fluid sugar in bacterial meningitis: another look. Pediatrics 1969, 44:1-3.

67. Paulson OB, Hansen EL, Kristensen HS, Brodersen P: Cerebral blood flow, cerebral metabolic rate of oxygen and CSF acid-base parameters in patients with acute pyogenic meningitis and with acute encephalitis. Acta Neurol Scand Suppl 1972, 51:407-408.

68. Posner JB, Plum F: Independence of blood and cerebrospinal fluid lactate. Arch Neurol 1967, 16:492-496.

69. Bosman J, Mourik I, Rasch Mv, Sieverts E, Verhoeff H: Scopus reviewed and compared. The coverage and functionality of the citation database Scopus, including comparisons with Web of Science and Google Scholar. Utrecht University Library 2006 [http://igitur-archive.library.uu.nl/ DARLIN/2006-1220-200432/Scopus\%20doorgelicht\%20\%26\%20vergeleken\% 20-\%20translated.pdf].

70. Sterne JA, Juni P, Schulz KF, Altman DG, Bartlett C, Egger M: Statistical methods for assessing the influence of study characteristics on treatment effects in 'meta-epidemiological' research. Stat Med 2002, 21:1513-1524.

doi:10.1186/cc9395

Cite this article as: Huy et al:: Cerebrospinal fluid lactate concentration to distinguish bacterial from aseptic meningitis: a systemic review and meta-analysis. Critical Care 2010 14:R240. 